\title{
ESTUDO DA TÉCNICA DE ANÁLISE ESTRUTURAL DE UMA PRENSA HIDRÁULICA VIA MÉTODO DE ELEMENTOS FINITOS (MEF)
}

\section{STUDY OF THE STRUCTURAL ANALYSIS TECHNIQUE OF A HYDRAULIC PRESS VIA FINITE ELEMENT METHOD (MEF)}

\author{
Alan Elson Queiroz \\ Igor Rocha dos Santos ${ }^{\text {II }}$ \\ Gustavo Coser Monteiro Dias ${ }^{\text {III }}$ \\ Maria Aparecida Bovério ${ }^{\mathrm{IV}}$ \\ Rogério Aparecido Miranda ${ }^{\mathrm{V}}$
}

\begin{abstract}
RESUMO
Este artigo apresenta os resultados de uma pesquisa, cuja ideia foi utilizar uma ferramenta de simulação para um projeto de uma estrutura de uma prensa de capacidade 5 toneladas, onde foi realizado um modelo desenvolvido no Software SolidWorks e utilizado a ferramenta simulation do programa que utiliza métodos de elementos finitos (MEF) para realizar diversos cálculos de um modelo qualquer, retornando resultados com tensão máxima, mínima no modelo, deslocamento máximo da estrutura após aplicação da carga, fator de segurança, entre outros. Nesse estudo o modelo foi simplificado para facilitar o processamento e diminuir tempos computacionais, atribuído material para o modelo, definido pontos de fixação, carga de solicitação e criado as malhas. Para isso, foram realizados experimentos para encontrar a melhor forma de otimizar o modelo para realizar os controles de malhas nas regiões mais importantes. Após toda parte experimental do trabalho, foi encontrada a melhor maneira de otimizar o modelo para se obter uma solução aproximada do ideal para o presente estudo e, para isso, utilizou-se de raios para eliminar as singularidades de tensão e de um aumento de espessura em parte do modelo para se obter através de um gráfico uma curvatura de tensão que tende a convergir com valores inferiores a tensão do limite de escoamento do material utilizado.
\end{abstract}

Palavras-chave: Elementos finitos. Malhas. Solução aproximada. Convergir.

\begin{abstract}
This article presents the results of a research, whose idea was to use a simulation tool for a design of a 5 ton capacity press structure, where a model developed in the SolidWorks

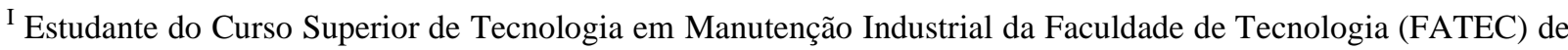
Sertãozinho - São Paulo - Brasil. E-mail: alan_queiroz2008@ hotmail.com.

II Estudante do Curso Superior de Tecnologia em Manutenção Industrial da Faculdade de Tecnologia (FATEC) de Sertãozinho - São Paulo - Brasil. E-mail: igor_rocha100@hotmail.com.

III Prof. Me. da Faculdade de Tecnologia (FATEC) de Sertãozinho - São Paulo - Brasil. E-mail: gustavo.dias10@fatec.sp.gov.br.

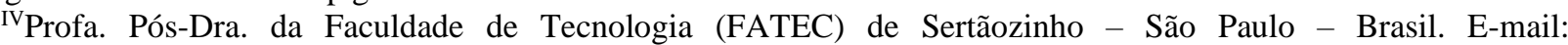
mariaboverio@hotmail.com.

vProf. Esp. da Faculdade de Tecnologia (FATEC) de Sertãozinho - São Paulo - Brasil. E-mail: rogerio.miranda@fatec.sp.gov.br.
} 


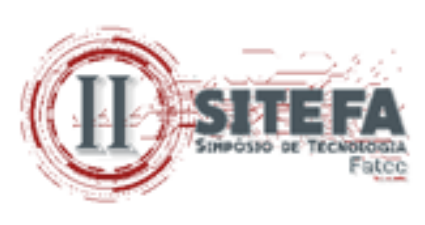

Software was performed and used the Simulation tool of the program that uses finite element methods (MEF) to perform several calculations of any model, returning results with maximum voltage, minimum in the model, maximum displacement of the structure after application of the load, factor of Security, among others. In this study the model was simplified to facilitate processing and decrease computational times, attributed material to the model, defined fixation points, request load and created the meshes. For this, experiments were carried out to find the best way to optimize the model to perform the controls of meshes in the most important regions. After all experimental part of the work, we found the best way to optimize the model to obtain an approximate solution of the ideal for the present study and, for this, we used rays to eliminate the singularities of tension and an increase in thickness In part of the model to obtain through a graph a tension curvature that tends to converge with values lower than the voltage of the flow limit of the material used.

Keywords: Finite elements. Meshes. Approximate solution. Converge.

Data de submissão do artigo: 15/07/2019.

Data de aprovação do artigo: 13/08/2019.

DOI:

\section{INTRODUÇÃO}

A possibilidade de realizar, via Método de Elementos Finitos (MEF), estudos do comportamento de máquinas e equipamentos durante funcionamento sob carregamento permitiu aos fabricantes de softwares do tipo Computed Aided Design (CAD), associarem o módulo de estudos via elementos finitos (Computed Aided Engineering, ou simplesmente $\mathrm{CAE}$ ) à ferramenta de projeto. Essa estratégia ofereceu aos engenheiros e projetistas uma maior versatilidade nos momentos de criação, dimensionamento e verificação dos projetos. Dentre as opções disponíveis no mercado e, por ser um software devidamente licenciado pela Faculdade de Tecnologia do Estado de São Paulo (FATEC), campus de Sertãozinho/SP, optou-se pelo SolidWorks para a realização deste trabalho.

O presente artigo tem como problema de pesquisa a elaboração de um estudo da segurança estrutural do projeto de uma prensa hidráulica de baixa capacidade (cinco toneladas de força), para aplicação em atividades acadêmicas. Este estudo foi realizado via análise por elementos finitos a partir de uma estrutura metálica também projetada em SolidWorks.

\section{SOFTWARE CAD/CAE}

A utilização de um software CAD/CAE permite elaborar e analisar o projeto mecânico de um equipamento mecânico utilizando-se de uma mesma ferramenta, com apreciável confiabilidade. Com este propósito, o SolidWorks tem sido uma ferramenta bastante empregada na indústria brasileira, justamente em razão da versatilidade, além de oferecer uma interface com o usuário bastante intuitiva (FIALHO, 2012)

Com o advento do recurso das análises estruturais via elementos finitos as empresas passaram a minimizar a utilização do procedimento de tentativas e erros (MAMALIS; MANOLAKOS; BALDOUKAS, 1996) e, consequentemente, reduziram os custos operacionais envolvidos com essa prática. Por outro lado, usuários inexperientes do Método de Elementos Finitos (MEF) podem sentir-se tentados a aceitar quaisquer resultados obtidos a 


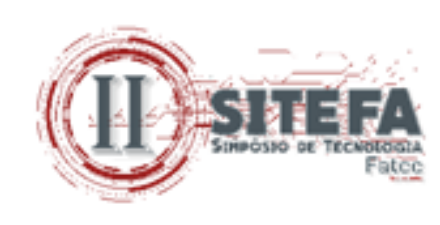

partir do modelo matemático empregado para a situação em estudo, unicamente por julgarem que o software escolhido é de extrema qualidade (AZEVEDO, 2003).

Uma vez que o MEF se utiliza de simplificações a partir de situações complexas reais, deve-se conhecer em profundidade o modelo matemático empregado antes de assumir os resultados obtidos como sendo reproduções fiéis da situação física real (AZEVEDO, 2003). A metodologia é bastante empregada em situações em que se busca reproduzir, ou simular o comportamento estrutural, térmico e ou de um fluido durante o escoamento. A solução de problemas práticos de engenharia exige o emprego de computadores digitais e a demanda por capacidade de processamento desses aumenta conforme o número de não linearidades envolvidas (AZEVEDO, 2003; BATHE, 2014).

A prensa hidráulica em questão foi idealizada e projetada para ser utilizada como ferramenta de apoio no preparo e realização de aulas práticas nos laboratórios da FATEC de Sertãozinho. Como matéria prima para a estrutura metálica da prensa optou-se pelo aço ASTM A-36 por combinar resistência mecânica (tensão de escoamento de $250 \mathrm{MPa}$ e resistência a tração de 400MPa) com resiliência (LUZ, 2019).

\section{PROCEDIMENTOS METODOLÓGICOS}

O trabalho começou a partir da idealização de uma prensa hidráulica de baixa capacidade de carga para empregá-la no preparo de aulas de laboratório da FATEC de Sertãozinho, além da realização de eventuais montagens e desmontagem de componentes mecânicos. Pensando nessas aplicações, o modelo estrutural de prensa proposto (Figura 1) permite o posicionamento da mesa de trabalho em até seis posições diferentes, conforme o comprimento da peça ou componente a ser trabalhada. As dimensões principais da prensa estão apresentadas na Figura 1, que oferece uma rápida percepção da forma construtiva do equipamento.

Figura 1 - Forma construtiva da prensa hidráulica
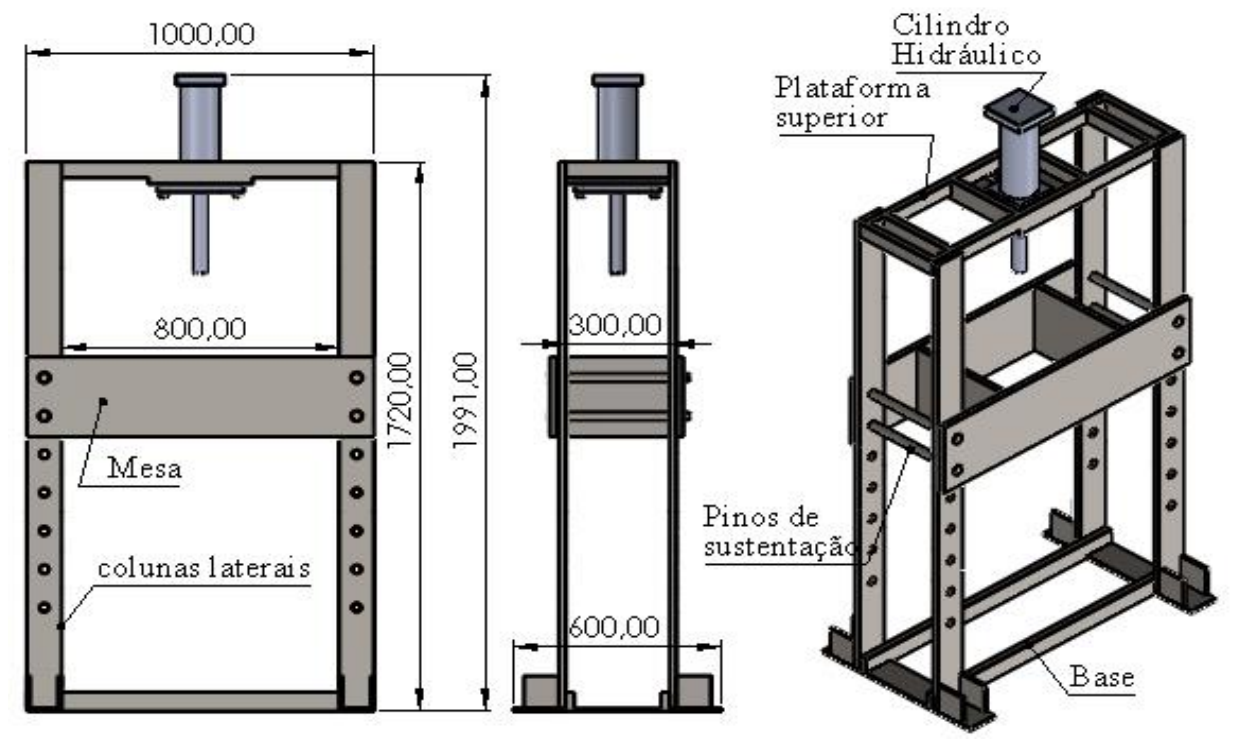

Fonte: autoria própria (2019) 


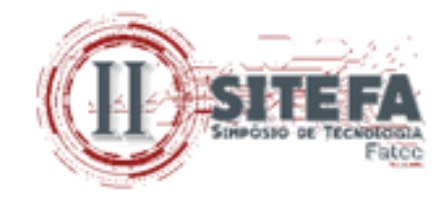

O modelo de prensa proposto é composto de uma mesa com regulagem de altura e fixação por meio de quatro pinos de sustentação, posicionados no interior de furos concêntricos. Sobre a mesa de trabalho apoiam-se as peças que receberão a força aplicada pelo cilindro. $\mathrm{O}$ equipamento está apoiado sobre o solo por meio de uma base de apoio e possui uma plataforma superior onde é instalado o cilindro hidráulico.

O projeto estrutural da prensa prevê a utilização de cordões de soda na fixação da plataforma superior às colunas laterais do equipamento. Por se tratar de uma região de elevada responsabilidade, optou-se por avaliar o coeficiente de segurança adotado no projeto para esse subconjunto. Para isso, e a fim de simplificar o modelo a ser empregado no MEF e reduzir o tempo computacional para convergência da solução, optou-se por trabalhar com a estrutura apresentada na Figura 2.

Foi adotada a ferramenta de análise simulation do software SolidWorks 2018 x64 Edition, devido a versatilidade oferecida de resolver vários problemas enquanto projeta uma determinada peça ou um equipamento, obtendo resultados como tensão máxima ou mínima na peça, fator de segurança e deslocamentos. O modelo projetado da estrutura da prensa hidráulica tem que suportar uma carga de 5 toneladas com coeficiente de segurança maior que 1,0. Para o MEF optou-se como material de confecção da estrutura o aço ASTM A-36 com um limite de escoamento de $250 \mathrm{~N} / \mathrm{mm}^{2}$ e adotou-se como elementos de fixação de geometria, os oito furos de sustentação da mesa de trabalho destacados na Figura 2. Aplicou-se uma carga distribuída de 5 toneladas $(49,03 \mathrm{KN})$ na região de fixação do cilindro hidráulico, cuja área é de $0,67 \mathrm{~m}^{2}$.

Figura 2 - Modelo empregado no estudo via MEF

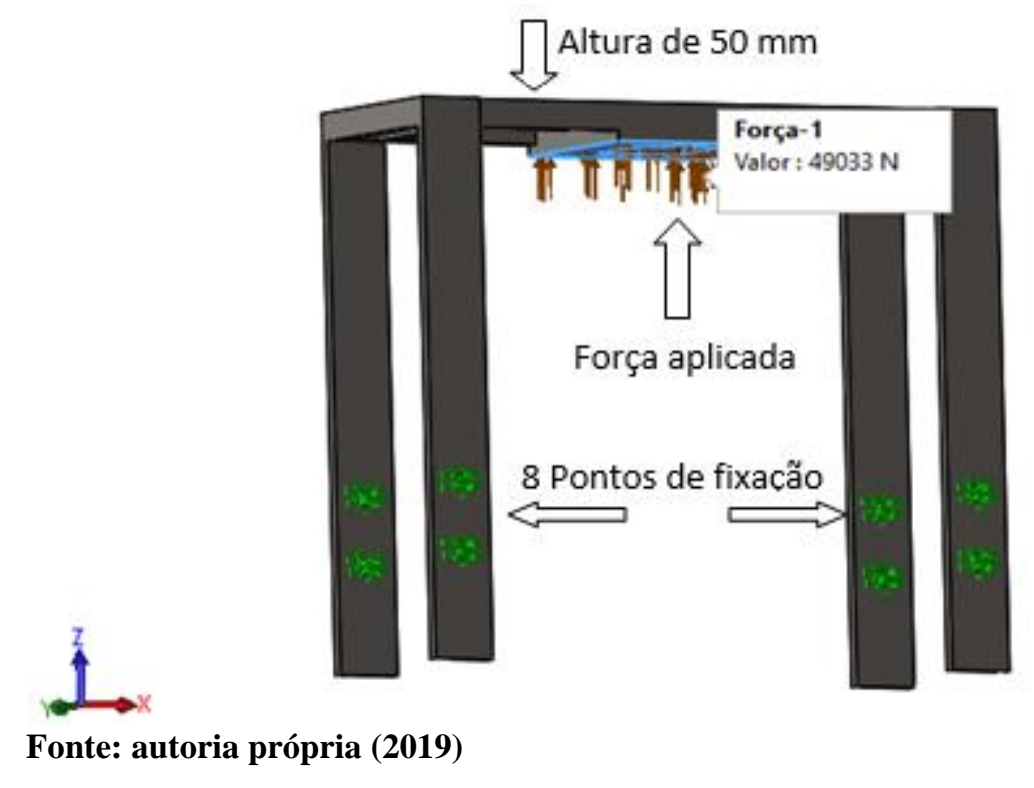

Em um primeiro estudo foi utilizada uma malha padrão em toda peça sem alterar nenhum parâmetro, com tamanho global de $24,902 \mathrm{~mm}$ e tolerância de $1,245 \mathrm{~mm}$ e 4 pontos jacobianos conforme representado pela Figura 3. 


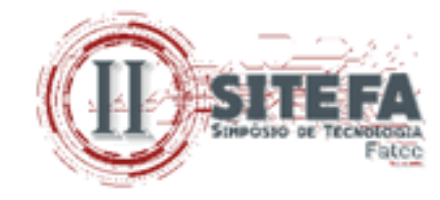

Figura 3 - Malha gerada para o primeiro estudo do modelo

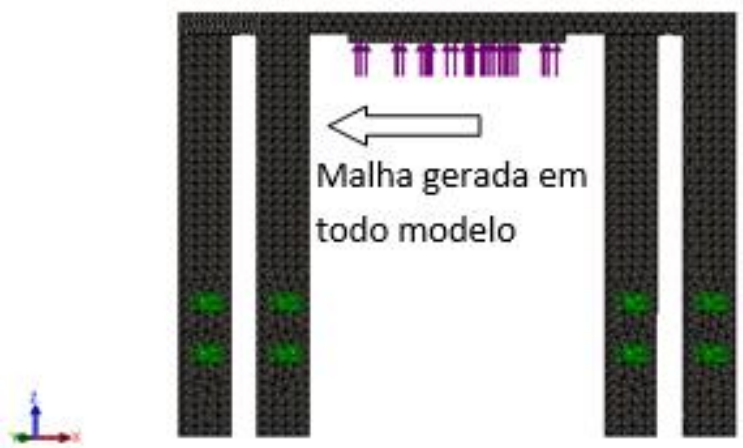

Fonte: autoria própria (2019)

\section{RESULTADOS E DISCUSSÃO}

Após execução do primeiro estudo, o resultado do fator de segurança do modelo é de 1,3 e a tensão Von Mises máxima no modelo de $192 \mathrm{MPa}$, conforme mostrado na Figura 4, que evidencia as quatro regiões mais críticas do modelo, que coincidem com os cordões de solda supramencionados.

Figura 4 - Análise estática de tensão pelo critério de Von Mises

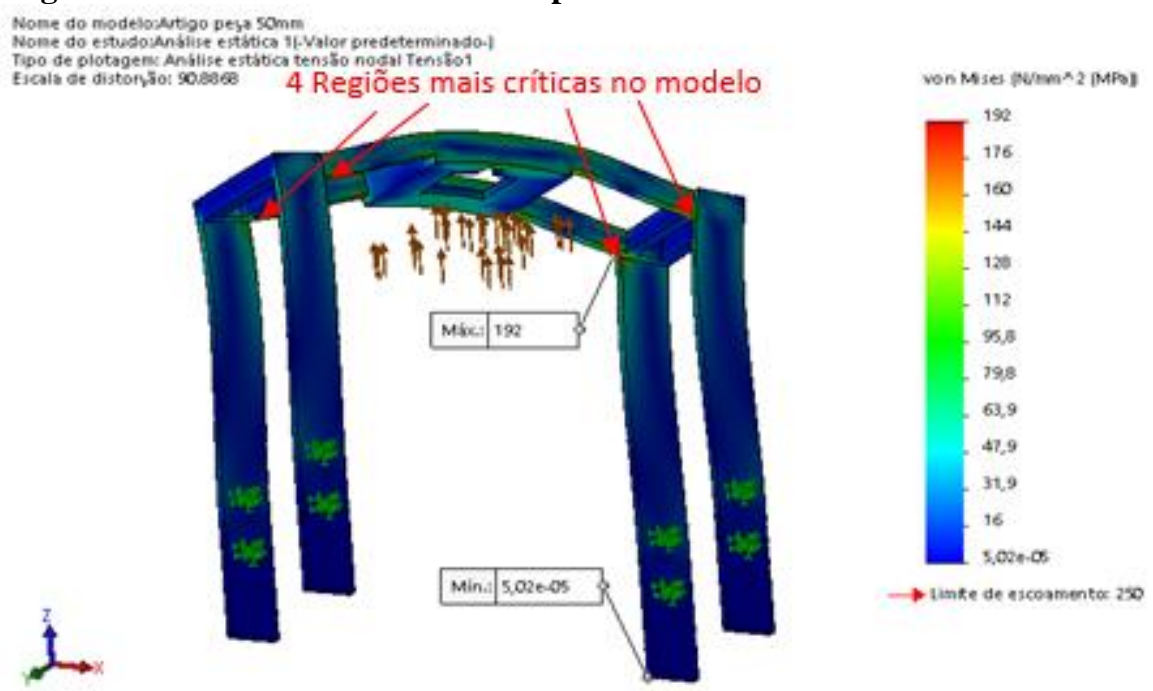

Fonte: autoria própria (2019)

Como resultado para o máximo deslocamento obtido registrou-se $1,1 \mathrm{~mm}$ de movimentação vertical ocorrida no centro da plataforma superior, conforme mostra a Figura 5. Os resultados apresentados nas Figuras 4 e 5 apresentam uma escala de distorção de 90.8863 vezes a movimentação real da estrutura e, por isso é visível a deformação. 


\section{(II) SITEFA}

Figura 5 - Análise do limite de deslocamento URES (mm)

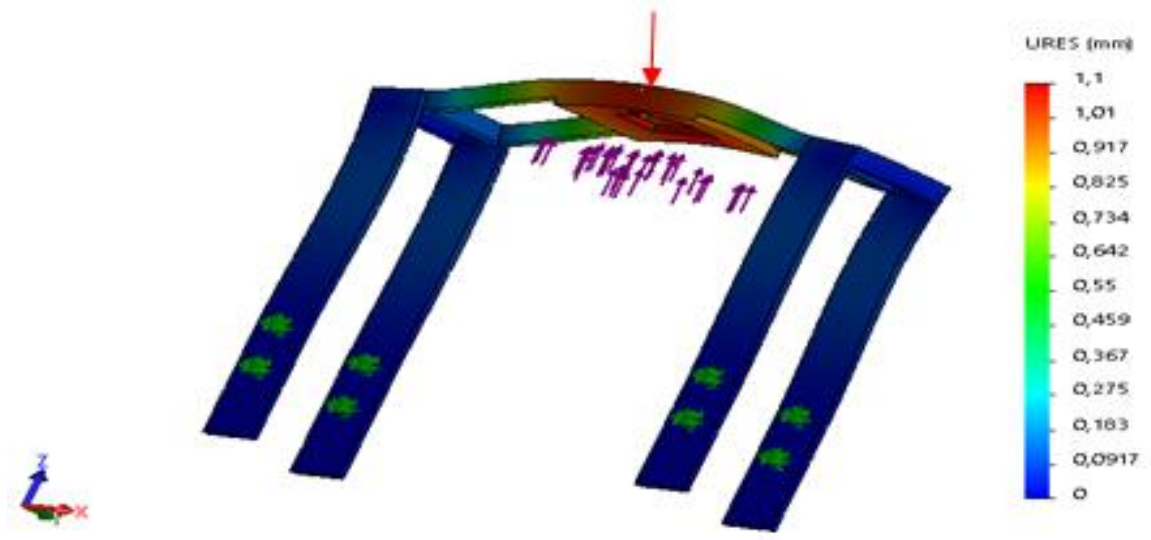

Fonte: autoria própria (2019)

O resultado preliminar trouxe o questionamento de qual deveria ser o tamanho mínimo dos elementos adotados de modo que o modelo convergisse para a solução com menor erro em relação à aplicação física. A fim de responder a esse questionamento optou-se por refinar a malha gradativamente. Durante essa atividade observou-se que a tensão Von Mises máxima no modelo aumentava, ultrapassando o limite de escoamento do material. O refinamento da malha para todo o modelo veio acompanhado de um aumento considerável do tempo de processamento, que passou de 10 segundos, para elementos de $25 \mathrm{~mm}$, aproximadamente, para 45 minutos para elementos de $2 \mathrm{~mm}$ de tamanho médio.

No intuito de manter o tempo de processamento dentro de um limite aceitável, próximo de 10 segundos originais, optou-se por refinar a malha apenas na região crítica do modelo, mantendo para o restante do modelo, uma malha mais grosseira. Esse recurso diminui o tempo de processamento do estudo e mantém o compromisso por resultados mais confiáveis que aquele apresentado pelo teste preliminar.

Para aplicar o controle de malha nas regiões desejadas, o método utilizado foi realizar ressaltos de $0,01 \mathrm{~mm}$ na região crítica no modelo, de modo a gerar superfícies de referência para a nova malha, conforme observado na Figura 6.

Figura 6 - Método utilizado para realizar controle de malhas

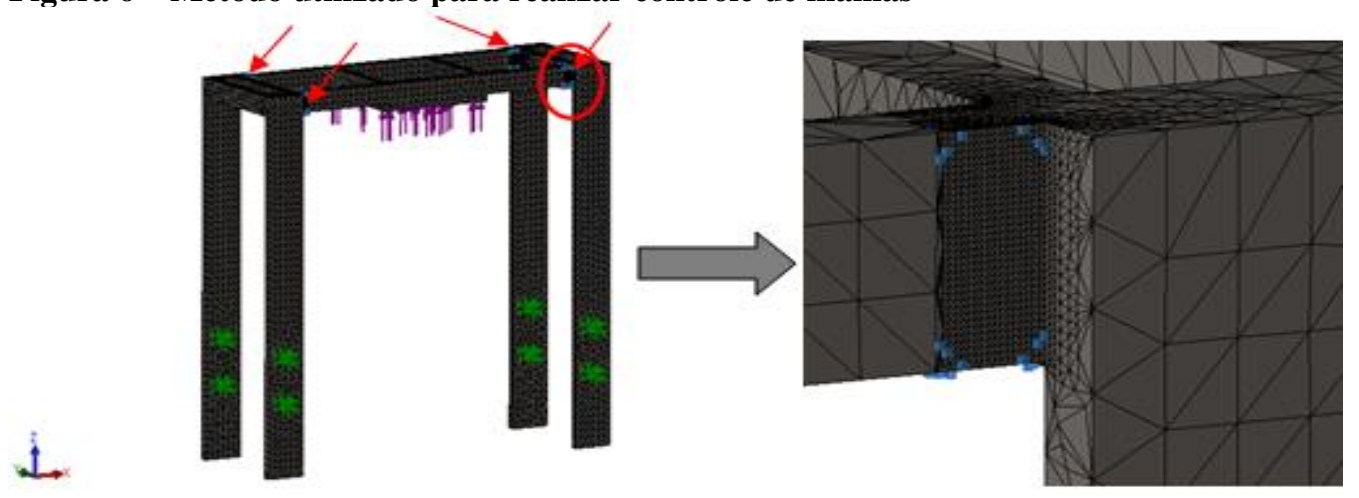

Fonte: autoria própria (2019)

Após o uso do recurso do ressalto, trabalhou-se com malhas de diversos tamanhos, conforme mostrado na Tabela 1. O Gráfico 1 mostra a evolução da máxima tensão de Von 


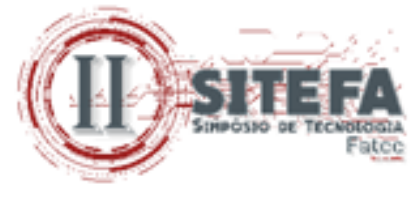

Mises conforme tamanho médio dos elementos empregados na malha correspondente às regiões críticas do modelo.

Tabela 1 - Tamanho dos elementos de malha utilizados e máxima tensão de Von Mises

\begin{tabular}{ccccc}
\hline \multicolumn{5}{c}{ TABELA DE MALHAS, TENSÕES E TEMPOS } \\
\hline ESTUDO & $\begin{array}{c}\text { MALHA } \\
(\mathbf{m m})\end{array}$ & $\begin{array}{c}\text { TENSÃO } \\
(\text { MPa) }\end{array}$ & $\begin{array}{c}\text { TEMPO DE } \\
\text { GERAÇÃO DA } \\
\text { MALHA (s) }\end{array}$ & $\begin{array}{c}\text { TEMPO DE } \\
\text { EXECUÇÃO DO } \\
\text { ESTUDO (s) }\end{array}$ \\
\hline 1 & 10 & 385 & 4 & 12 \\
2 & 8 & 402 & 4 & 13 \\
3 & 6 & 457 & 4 & 14 \\
4 & 4 & 564 & 5 & 15 \\
5 & 2 & 807 & 8 & 60 \\
6 & 1,5 & 889 & 16 & 6 \\
\hline
\end{tabular}

Fonte: autoria própria. (2019)

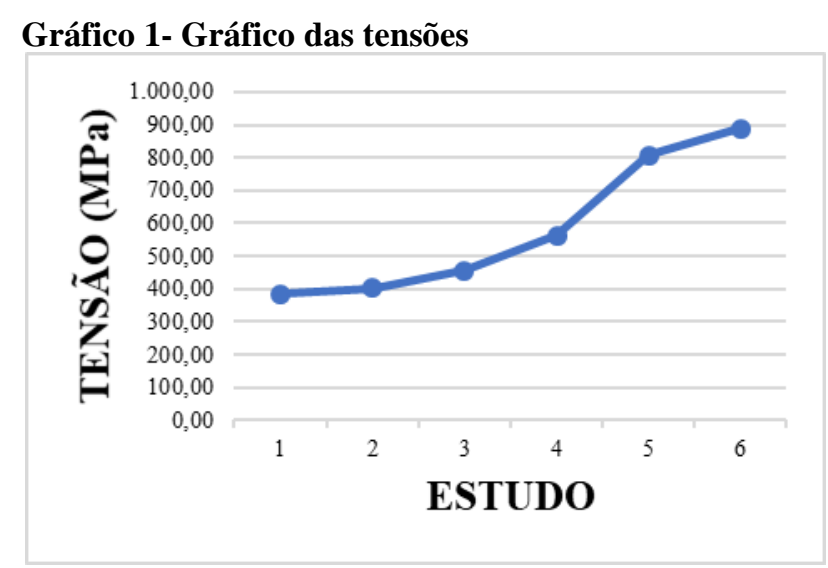

Fonte: autoria própria. (2019)

O intuito do trabalho de refino de malha foi observar a curva e identificar a malha ideal para o modelo, contudo, conforme reduziu-se o tamanho da malha observou-se que a tensão aumentava, constatando que o modelo não suportaria a carga solicitada. Uma vez entendido que o modelo continha um problema de dimensionamento nas chapas que compunham a plataforma, aumentou-se a altura destas para $80 \mathrm{~mm}$ e depois para $100 \mathrm{~mm}$, a fim de verificar a altura ideal.

O trabalho de refino de malha foi reproduzido para as duas novas alturas de plataforma e observou-se que ao diminuir a malha de $4 \mathrm{~mm}$ para $2 \mathrm{~mm}$ de tamanho médio dos elementos, a curva ultrapassava o valor do limite de escoamento nas mesmas regiões críticas. Esse resultado contrariava o comportamento esperado de aumento na resistência mecânica do conjunto. Observou-se, portanto, que ao criar ressaltos de $0,01 \mathrm{~mm}$ para aplicar o controle de malhas, o modelo estava criando vértices no ressalto, gerando ainda mais concentradores de tensão nessas regiões o que invalidava os resultados.

A solução proposta para mitigar o problema dos concentradores de tensão, imposto pelos ressaltos, foi de aplicar raios de $6 \mathrm{~mm}$ em todos os vértices nas regiões críticas, conforme mostra a Figura 7. 


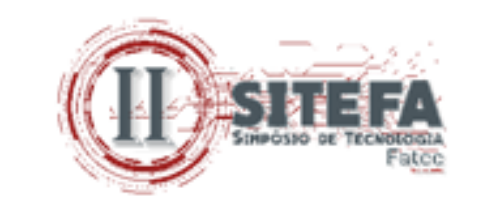

Figura 7 - Otimização com raios e controle de malhas nos raios

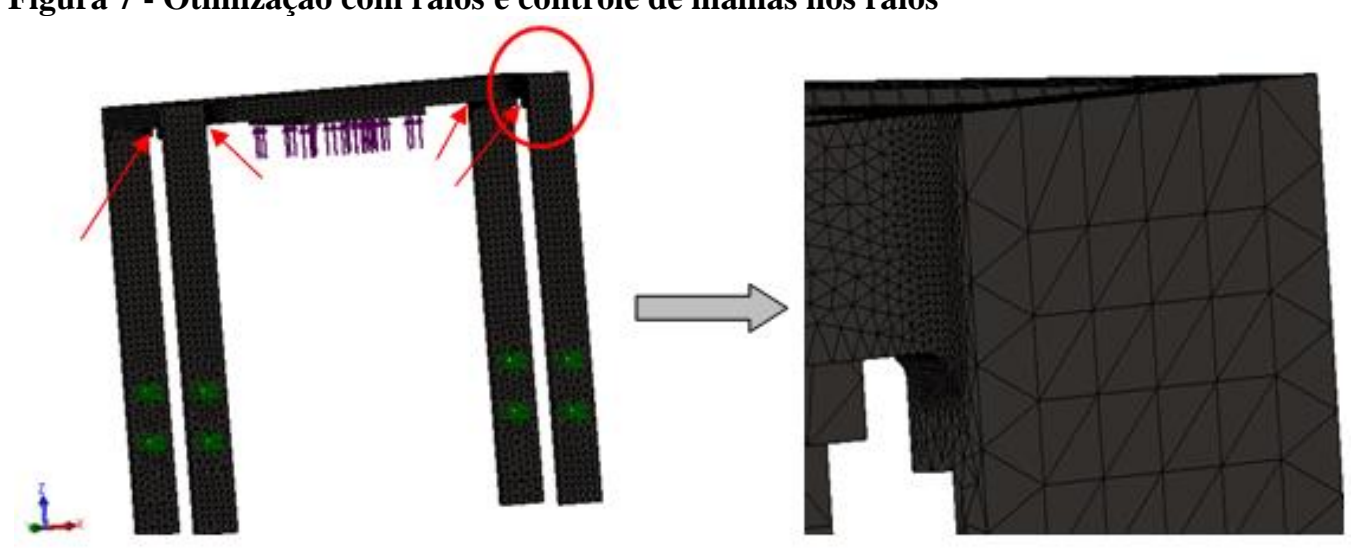

Fonte: autoria própria (2019)

Após gerados os raios de curvatura aplicou-se o controle de malha e repetiu-se o estudo com as mesmas malhas adotadas na Tabela 1. Os novos resultados obtidos são apresentados na Tabela 2, cuja curva de convergência está plotada no Gráfico 2. Observou-se que no primeiro estudo com ressaltos a diferença de tensão utilizada no controle de malha de $10 \mathrm{~mm}$ para $1,5 \mathrm{~mm}$ foi de $504 \mathrm{MPa}$. A reprodução deste mesmo estudo, porém com os raios nas extremidades derrubou essa diferença para $98 \mathrm{MPa}$, o que comprova que a criação dos ressaltos para fazer controle de malhas acrescenta concentradores de tensão, que são indesejados e camuflam os resultados.

Tabela 2 - Tabela de malhas utilizadas e tensões obtidas configuração com raios

\begin{tabular}{ccccc}
\hline \multicolumn{5}{c}{ TABELA DE MALHAS, TENSÕES E TEMPOS } \\
\hline ESTUDO & $\begin{array}{c}\text { MALHA } \\
(\mathbf{m m})\end{array}$ & $\begin{array}{c}\text { TENSÃO } \\
(\text { MPa })\end{array}$ & $\begin{array}{c}\text { TEMPO DE } \\
\text { GERAÇÃO DA } \\
\text { MALHA (s) }\end{array}$ & $\begin{array}{c}\text { TEMPO DE } \\
\text { EXECUÇÃO DO } \\
\text { ESTUDO (s) }\end{array}$ \\
\hline 1 & 10 & 334 & 4 & 12 \\
2 & 8 & 352 & 5 & 13 \\
3 & 6 & 360 & 5 & 14 \\
4 & 4 & 387 & 5 & 16 \\
5 & 2 & 412 & 7 & 29 \\
6 & 1,5 & 432 & 11 & 55 \\
\hline
\end{tabular}

Fonte: autoria própria. (2019) 


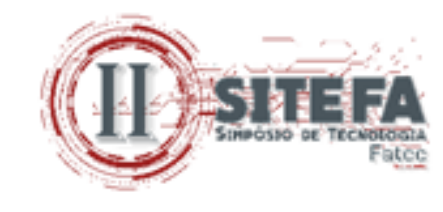

Gráfico 2- Gráfico das tensões

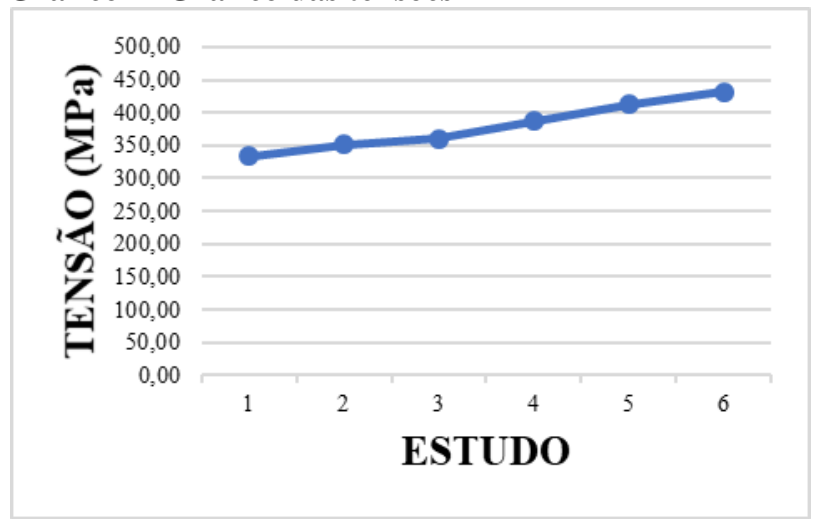

Fonte: autoria própria. (2019)

O Gráfico 2 mostra que a curva tende a convergir conforme se refina as malhas, mas como o limite de escoamento do material é de $250 \mathrm{MPa}$ é necessário otimizar o modelo alterando material com limite de escoamento maior ou aumentar altura da plataforma superior e refazer os estudos até a curva convergir dentro do limite de escoamento do material utilizado para o modelo. Nesse estudo a alternativa foi aumentar a altura da plataforma superior para $80 \mathrm{~mm}$.

Após a realização de todo estudo para encontrar a forma de otimizar o modelo e realizar os controles de malhas adequadamente, definiu-se uma altura para plataforma superior de $80 \mathrm{~mm}$ com raios de $6 \mathrm{~mm}$ nos vértices onde há maiores solicitações de tensão, conforme Figura 8.

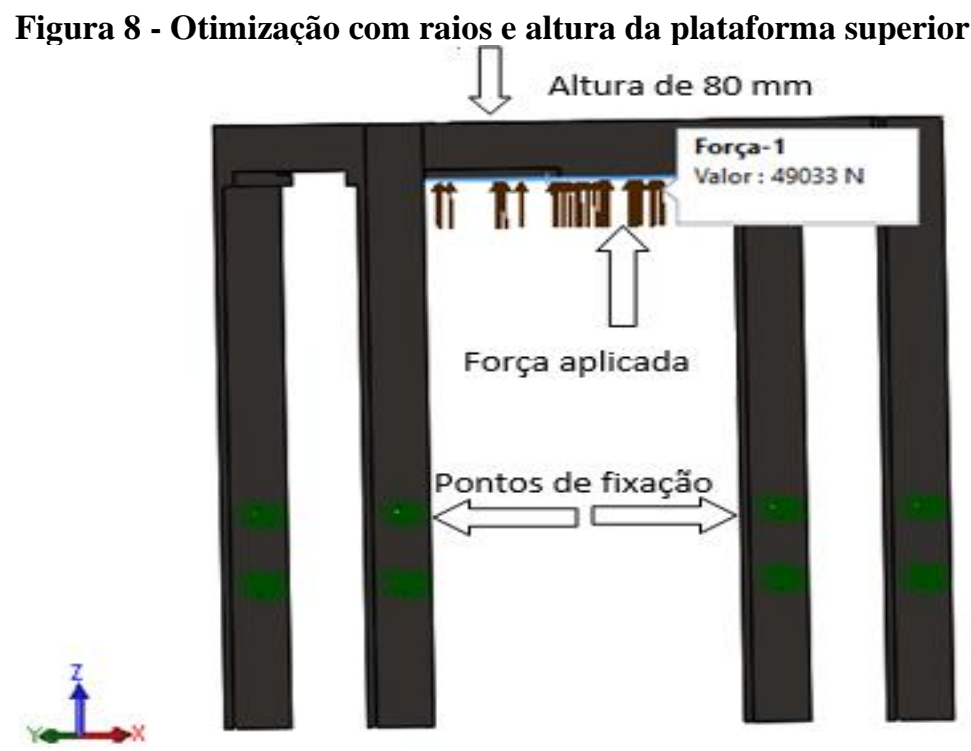

Fonte: autoria própria (2019)

A Tabela 3 traz os dados de tamanho médio dos elementos de malha e máximas tensões de Von Mises para cada estudo realizado com altura de $80 \mathrm{~mm}$ e raios de $6 \mathrm{~mm}$. O Gráfico 3 oferece a curva de convergência para essa mesma condição. 


\section{(iii) SIIEEA}

Tabela 3 - Tabela de malhas utilizadas e tensões obtidas configuração com raios altura 80mm.

\begin{tabular}{ccccc}
\hline \multicolumn{5}{c}{ TABELA DE MALHAS, TENSÕES E TEMPOS } \\
\hline ESTUDO & $\begin{array}{c}\text { MALHA } \\
(\mathbf{m m})\end{array}$ & $\begin{array}{c}\text { TENSÃO } \\
(\text { MPa) }\end{array}$ & $\begin{array}{c}\text { TEMPO DE } \\
\text { GERAÇÃO DA } \\
\text { MALHA (s) }\end{array}$ & $\begin{array}{c}\text { TEMPO DE } \\
\text { EXECUÇÃO DO } \\
\text { ESTUDO (s) }\end{array}$ \\
\hline 1 & 10 & 151 & 3 & 12 \\
2 & 8 & 147 & 4 & 13 \\
3 & 6 & 154 & 5 & 15 \\
4 & 4 & 162 & 5 & 18 \\
5 & 2 & 173 & 12 & 37 \\
6 & 1,5 & 181 & 18 & 59 \\
\hline
\end{tabular}

Fonte: autoria própria (2019)

Gráfico 3- Gráfico das tensões

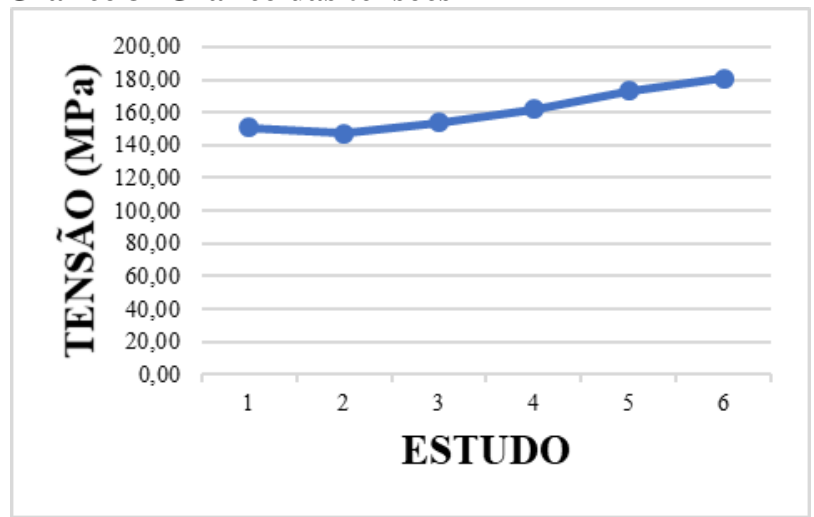

Fonte: autoria própria (2019)

Observa-se no Gráfico 3 que a curva tende a convergir conforme refinamento da malha a níveis de tensão abaixo do limite de escoamento do material. Para o fator de segurança pode-se verificar que o item "estudo 6" da Tabela 3 registrou 1,38 e para o máximo deslocamento vertical, 0,46 $\mathrm{mm}$ no ponto central da plataforma superior.

\section{CONCLUSÃO}

Os resultados obtidos nessa pesquisa através do software são resultados aproximados, pois há uma série de fatores implicando na solução do modelo, como: aproximação do método matemático que o próprio software realiza, simplificações de condições de restrições que se referem à maneira que o usuário simplifica o modelo, a citar a forma em que se fixa o modelo para aplicação da carga, simplificações das cargas aplicadas, simplificação do modelo de material, uma vez que o programa não considera possíveis descontinuidades que possam existir no material, entre outras. Essas simplificações são necessárias para solucionar análises por método de elementos finitos e, por isso, podemos afirmar que os resultados são aproximados. Com o SolidWorks foi possível projetar, simular e melhorar a estrutura mecânica da prensa hidráulica, que passou de uma altura de $50 \mathrm{~mm}$ para $80 \mathrm{~mm}$.

O ponto de maior dificuldade foi eliminar as singularidades de tensões, que estavam retornando resultados muito insatisfatórios. A solução de utilizar os raios nos pontos críticos 


\section{(iil) $\sin$ EA}

foi suficiente para obter melhores resultados, que permitiram definir um modelo aproximado que possa satisfazer a fabricação de um protótipo do equipamento.

Este trabalho pode contribuir para novos estudos que envolvam simulações com o software SolidWorks, uma vez que essa pesquisa mostrou uma forma de otimizar o modelo de estudos via MEF.

\section{REFERÊNCIAS}

AZEVEDO, A. Método dos elementos finitos. 2003. Disponível em:

http://alvaroazevedo.com/publications/books/Livro_MEF_AA_1ed/doc/Livro_MEF_AA.pdf. Acesso em: 15 de jun. 2019.

BATHE, K., J. Finite Element Procedures. 2 ed. Watertown: Prentice Hall, Pearson Education, Inc., 2014. 1065 p.

FIALHO, A. B. SolidWorks Premium. 2012: teoria e prática no desenvolvimento de produtos industriais-plataforma para projeyos CAD/CAE/CAM. São Paulo: Érica Ltda.

LUZ, Gelson. Blog materiais. 2019. Disponível em:

https://www.materiais.gelsonluz.com/2017/10/astm-a36-propriedades-mecanicas-ecomposicao-quimica.html. Acesso em: 15 jun. 2019.

MAMALIS, A., G., MANOLAKOS, D., E., BALDOUKAS, A., K., On the finite-element modelling of the deep-drawing of square sections of coated steels. Journal of Materials Processing Technology, v. 58, p. 153-159, 1996. 